\title{
EL POTENCIAL EMPRENDEDOR DEL ESTUDIANTE UNIVERSITARIO: CASO FACULTAD DE CIENCIAS ECONÓMICAS, ADMINISTRATIVAS Y CONTABLES DE LA UNAH, 2019, PRIMERA FASE
}

\author{
Jesús David Argueta Moreno*
}

DOI: $10.5377 /$ eya.v10i2.9087

Recibido: 25/9/2019 Aceptado: 20/12/2019

\section{RESUMEN}

La presente investigación, compiló insumos, en torno a la caracterización del Potencial Emprendedor del estudiante de la Facultad de Ciencias Económicas, Administrativas y Contables (FCEAC) de la Universidad Nacional Autónoma de Honduras (UNAH), Campus Ciudad Universitaria en Tegucigalpa, con el objetivo de conocer los factores intrínsecos y extrínsecos que fortalecen y/o limitan el potencial/capacidad emprendedora de los jóvenes estudiantes de esta facultad. De igual forma, se exploraron las principales habilidades, competencias y pericias de los estudiantes, que atañen a su capacidad emprendedora. Cabe mencionar que este análisis representa la primera fase del análisis del potencial emprendedor de las y los estudiantes de la facultad, considerando que se desarrollarán futuras pesquisas que ampliarán el entendimiento de este fenómeno.

Palabras claves: emprendimiento, habilidades, competencias, pericias, conocimientos, administración del riesgo

JEL: L26, J24

\footnotetext{
* Profesor del Departamento de Administración de Empresas de la Facultad de Ciencias Económicas, Administrativas y Contables de la Universidad Nacional Autónoma de Honduras (FCEAC-UNAH). Correo electrónico: jesus.argueta@unah.edu.hn
} 


\title{
THE UNIVERSITY STUDENT'S POTENTIAL ENTREPRENEUR: CASE FACULTY OF ECONOMIC, ADMINISTRATIVE AND ACCOUNTING SCIENCES OF THE UNAH, 2019, FIRST PHASE
}

Jesús David Argueta Moreno

DOI: $10.5377 /$ eya.v10i2.9087

\begin{abstract}
ABSRACT
This research collected inputs to characterize the entrepenuerial potencial of the students of the Faculty of Economic, Administrative and Accounting Sciences of the National Autonomous University of Honduras, Campus in Tegucigalpa (FCEAC-UNAH). The objective of this analysis is to understand the inner and outer factors that stimuli or conditioned the entrepreneurship skills and attitudes. This investigation represents the first stage of an overall research, among the entrepreneurship potential theme, across the undergraduate university scenario. Thereby, this study, evaluates the Core/Main Skills, Abilities, Knowledge and Entrepreneur Capacity.
\end{abstract}

Key Words: entrepreneur, skills, abilities, areas of competence knowledge, risk management.

JEL: L26, J24 


\section{INTRODUCCIÓN}

Este trabajo representa la primera fase de un cúmulo de investigaciones por realizar, en materia del análisis del potencial emprendedor de los estudiantes de la Facultad de Ciencias Económicas, Administrativas y Contables de la Universidad Nacional Autónoma de Honduras, en Ciudad Universitaria en Tegucigalpa (FCEAC-UNAH), siendo esta primera edición, un abordaje de tipo cualitativo, que permitió diagnosticar $y$ caracterizar las condiciones emprendedoras de las y los estudiantes en mención. Denotando que, a lo largo de este escrito, se describen varios elementos que directa e indirectamente, alteran el potencial emprendedor del universitario contemporáneo (ya sea de manera negativa o positiva). Quiere responder a la pregunta de investigación general de ¿cuáles son las principales características que definen el Potencial Emprendedor del Universitario?

Por lo expuesto con antelación, cabe mencionar que luego de cerrar esta entrega, se realizará una segunda fase, en marcada siempre en el tópico del Potencial Emprendedor Universitario, desde una óptica cuantitativa, demostrando posibles incidencias/correlaciones entre las variables aptitud emprendedora, actitud emprendedora y Condiciones Sociodemográficas, sobre el Potencial Emprendedor, partiendo del modelo propuesto por el académico Roberto Olmos (Olmos, 2011).

Por otro lado, es imperativo el destacar que, este análisis, tuvo la oportunidad de acercarse al alumnado de la Facultad, para levantar insumos de primera mano, a efecto de conocer sus limitantes, fortalezas, ópticas y tendencias hacia emprendimiento.

De igual manera, a lo largo de este análisis, se recaban varios insumos de fuentes secundarias, que permiten contextualizar el escenario emprendedor, de los jóvenes estudiantes de la Facultad, para contrastar la óptica de ellas(os), con un entorno que avizora ciertos riesgos de inversión y así, poder articular conjeturas que permitan a las autoridades universitarias, replantear estrategias de formación emprendedora, que se ajusten tanto a la realidad empresarial actual, como las expectativas emprendedoras de los estudiantes.

Por consiguiente, al adentrarse en el ámbito del emprendedurismo, es válido inferir que es sin lugar a duda, una tendencia, que se viene estudiando e impulsando, desde épocas recientes, por las administraciones estatales y grandes corporaciones, con el objetivo de mitigar el fenómeno del desempleo y estimular la creación de riqueza, desde una óptica macroeconómica.

Sin embargo, varios académicos que se han adentrado en el mundo del emprendimiento 
en América Latina, han demostrado en sus compilaciones empíricas y teóricas, que más del $90 \%$ de las empresas en el continente, son empresas familiares, independientemente de su tamaño (grande, pequeño, mediana o micro), validando la propuesta que el emprendedurismo en su versión sostenible, debe y puede convivir con varios actores y factores, que puedan estimular una simbiosis/equilibrio, entre los tiempos de su incubación, crecimiento y desarrollo (GEM, 2019).

El fenómeno del desempleo en el país se reproduce en medidas desproporcionadas, acentuándose día a día, con la inflación, reducción de la inversión nacional y extranjera, medidas erradas de "estimulo" a la empleabilidad, entre ellas: la ley del empleo por hora, la cual, ha demostrado ser poco efectiva en la calidad de vida de los ciudadanos en el país.

Por otro lado, diversos estudios realizados por Danilo A. David (Unidad Investigativa Diario la Prensa), y Enrique Posadas ( Gestor de Emprendimiento de la Universidad Tecnológica de Honduras) han demostrado que en la actualidad, de un total de 2,556,687 jóvenes hondureños, en la categoría de "millennial” y Generación “ $Z$ ”, solamente el $62 \%$ de ellas(os) tienen algún tipo de ingreso, producto de su trabajo en la empresa privada, gobierno y/o negocio propio; devengan menos de 15,000.00 Lempiras (David \& Posada, 2019).

Y para aquellas(os) millennials que tienen la oportunidad de abandonar el país, los cuales representan el $28 \%$ de la masa poblacional, previamente definida, se marchan, en busca de otras instancias laborales, la cual suele ir acompañada de condiciones de vida superiores, los cuales suelen buscarse regularmente en Norte América y varios países de Europa, dando paso al fenómeno de fuga de cerebros (David \& Posada, 2019).

Es por lo expuesto con antelación, que el masivo éxodo de hondureños no es más que el reflejo del deterioro en las condiciones de empleo, seguridad, salud, educación y de inversión, que en el país se experimenta. Estas condiciones, demandan al estudiante de educación superior, a adquirir más y nuevos conocimientos en su área de "expertise", al igual que en otros campos (FOSDEH, 2018). Es así, que la difícil tarea de competir en un escenario con tan pocos espacios laborales invita y obliga, al estudiante de la FCEACUNAH a buscar la mejora continua, robustecer sus habilidades, pericias $y$ competencias.

Señalando también, que del $100 \%$ de los jóvenes millennial hondureños, solamente el $34 \%$ de ellas(os) cursó sus estudios de bachillerato, 22\% de ellas(os) cuenta con una carrera universitaria, así mismo de este porcentaje, solamente el $11 \%$ de ellas(os) 
poseen una carrera técnica, enunciando algunas estadísticas, que exponen algunas de las causales del desempleo en el país, así como la dificultad en la temprana inserción en el mercado laboral local. Situación que les obliga a recorrer el riesgoso camino, hacia el emprendimiento por necesidad, lamentablemente, no por oportunidad, el cual no sugiere para la gran mayoría de estos modelos de negocio, un destino muy prometedor (COHEP, 2019).

Al mapear el área laboral, donde se encuentran los millennials hondureños, es válido mencionar que el $38 \%$ trabaja en la empresa privada, $21 \%$ estudia, $17 \%$ de ellas(os) trabaja en un negocio familiar/propio, 8\% labora en el Gobierno y un $17.7 \%$ de estos jóvenes, pertenece a la categoría de NINI's (es decir que Ni Estudian, Ni Trabajan), situación que agudiza, las condiciones económicas del país, al mencionar que $41 \%$ de las(os) jóvenes en mención, tienen hijos. Indicadores que describen a una población, altamente vulnerable a emigrar (COHEP, 2019). La situación aquí mencionada, dibuja un plano laboral complejo, al demandar altas habilidades, competencias, conocimientos y pericias, en un contexto de mucha incertidumbre y pocos espacios para albergar a los nuevos profesionales, situación que invita a estimular el emprendimiento entre los estudiantes de Educación Superior, de pre y posgrado.
El objetivo general de esta investigación es conocer las principales características que definen el potencial del emprendedor universitario. Como objetivos específicos interesa conocer cuáles son las principales características que definen el potencial emprendedor, sus condicionantes (endógenas y exógenas al entorno del estudiante), los factores que potencian $y$ estimulan, como también las fortalezas $y$ puntos a robustecer a nivel de conocimientos y competencias.

\section{MARCO TEÓRICO Y REFERENCIAL}

Previo a la exploración integral del sustento teórico y empírico, en la temática del potencial emprendedor del estudiante universitario. Es vital el familiarizar al lector con dos insumos claves para el pleno entendimiento del fenómeno a evaluar:

Los actores y variables, que intervienen en los procesos de formación y desarrollo de las aptitudes y actitudes emprendedoras de las y los estudiantes universitarios, como ser:

- Actitud Emprendedora.

- Aptitud Emprendedora.

- Las condiciones sociodemográficas que se manifiestan sobre los estudiantes universitarios.

En el caso de las Actitudes Emprendedoras, Ricardo Cabana, Iván Cortes y P. Robbinson, 
le conciben como la predisposición a responder de forma proactiva, a un determinado acontecimiento, al representar el principal componente del perfil de un emprendedor (Robbinson, 1991).

De igual manera el concepto de aptitud emprendedora, ha sido abordado por un sin número de académicos e industriales, entre los que destacan los aportes de J. Venecian y Roberto Olmos, al describirle como el conjunto de habilidades y pericias, que el emprendedor/profesional/técnico puede aprender en un marco temporal definido, robusteciendo sus habilidades primarias, secundarias y terciarias para emprender (Venecian, 1989).

Luego de explorar los términos de aptitud y actitud emprendedoras, es válido el hacer mención del término de capacidad emprendedora (siendo este el cúmulo aptitudes, actitudes y condiciones sociodemográficas de un individuo), el cual no es de origen reciente, pues desde 1924, S. Adler, lo define como un proceso de valoración y aprobación de varias condiciones que estimulan el potencial emprendedor, de un individuo, al ejercitar sus habilidades de autocrítica y reconocimiento de sus fortalezas $y$ debilidades afectivas, cognitivas $y$ profesionales, para iniciar con un modelo de negocios especifico (Adler, 1924).
Es así como, en América Latina, según Catherine Krauss, el potencial emprendedor, es el resultado de un conjunto de factores aptitudinales, actitudinales $y$ sociodemográficos, capaces de generar crecimiento social, así como de país, el cual depende estrictamente del conocimiento y experiencias acumuladas en el campo/rubro a emprender (Krauss, 2011).

En apoyo a lo mencionado, Humberto Sparano Rada, en varios de sus trabajos, define el emprendimiento como un proceso que con el transcurso del tiempo provoca alteraciones positivas en la creación de empresas, generando empleos, mejorando su eficiencia en la productividad, hasta lograr un nivel de competitividad en mercados nacionales e internacionales (Sparano, 2014). María Antonia Cervilla, también se ha sumado a la pesquisa del potencial emprendedor y emprendedurismo, certificando la labor de Humberto Sparano, debido a que en sus trabajos se describe al emprendimiento ("entrepreneurship") como el "proceso llevado a cabo por un individuo, para crear algo nuevo que agregue valor, asignando el tiempo y el esfuerzo necesarios, asumiendo los riesgos financieros, sociales y psicológicos asociados, y recibiendo la recompensa resultante en términos monetarios, de satisfacción personal y de independencia” (Cervilla \& Puente, 2013). 
Finalmente, otra de las definiciones, que mayor aceptación han reflejado en el ámbito académico del emprendedor, es la formulada por el Global Enterpreneurship Monitor (GEM), al ser una organización que monitorea de manera permanente el fenómeno del emprendimiento en el globo, define al emprendedor como una persona capaz de percibir una oportunidad y asumir los riesgos existentes para crear nuevos mercados, diseñar nuevos productos y desarrollar procesos innovadores (GEM, 2019).

Señalando que el emprendimiento, es estudiado tanto por académicos, como por industriales, que han creado asociaciones público-privadas para estudiar el fenómeno mismo, entre las cuales se destaca el Global Entepreneurship Monitor (GEM), el cual, es el esfuerzo más grande a nivel mundial para la medición del emprendimiento y la identificación de los factores que, a nivel institucional, cultural, normativo y de políticas públicas, determinan el nivel de actividad emprendedora, teniendo sus inicios en el año 2009, en la ciudad de Guatemala, por un equipo de profesionales y técnicos, especializados en la temática, provenientes del Centro de Emprendimiento Kirzner y de la Facultad de Ciencias Económicas de la Universidad Francisco Marroquín. De igual forma, el indicador GEM, se basa en la Encuesta Nacional sobre la participación y Actitudes hacia el Emprendimiento (Adult
Population Survey, APS), que mide el emprendimiento en la población adulta y la Encuesta Nacional de Expertos (National Expert Survey (NES)), que captura las percepciones de expertos acerca de entorno institucional y cultural dentro del cual se desarrolla los emprendimientos (GEM, 2019).

A continuación, se mencionan algunos de los principales ponentes en el marco de la temática del Potencial Emprendedor y sus dimensiones y/o tipologías, favor revisar la Tabla 1 a continuación:

Tabla 1. Compilación Teórica sobre los Valores que Acompañan la Actitud Emprendedora

\begin{tabular}{|l|c|}
\hline \multicolumn{1}{|c|}{ Autores } & $\begin{array}{c}\text { Tipologías/Dimensiones de } \\
\text { Emprendimiento según varios } \\
\text { Académicos }\end{array}$ \\
\hline $\begin{array}{l}\text { Bales y Couch } \\
(1969)\end{array}$ & $\begin{array}{c}\text { Autoridad, Auto Control, Equidad, } \\
\text { Individualismo }\end{array}$ \\
\hline Musek (1993) & $\begin{array}{c}\text { Hedonismo, Moral, Logro, } \\
\text { Cumplimiento }\end{array}$ \\
\hline $\begin{array}{l}\text { Rokeach } \\
(1973)\end{array}$ & Honestidad, Auto cumplimiento \\
\hline $\begin{array}{l}\text { Hofstede y } \\
\text { Bond (1984) }\end{array}$ & $\begin{array}{c}\text { Individualismo-Colectivismo, } \\
\text { Masculinidad-Feminidad, Evitar } \\
\text { Incertidumbre, Distancia del Poder }\end{array}$ \\
\hline $\begin{array}{l}\text { Schwartz } \\
(1992)\end{array}$ & $\begin{array}{c}\text { Apertura al Cambio, Conservación, } \\
\text { Auto trascender, Auto } \\
\text { engrandecimiento }\end{array}$ \\
\hline
\end{tabular}

Fuente: Morris et. Al (2002)

La Tabla 1, hace mención de algunos, de los principales ponentes en materia del análisis del potencial emprendedor, cuyos aportes, fueron en su momento articulados por un mismo modelo, realizado por Roberto 
Olmos, con el objetivo de medir el nivel potencial emprendedor de los estudiantes, abordándole como una variable de tipo longitudinal representado en la Figura 1, al describir el modelo en mención, para determinar el potencial emprendedor universitario, el cual fue validado y aplicado sobre la masa población universitaria de la Universidad Complutense de Madrid.

Señalando que el modelo base a adoptar por esta pesquisa, es el propuesto por Roberto Olmos, el cual, llega a parametrizar las variables independientes y dependientes (4 variables): aptitudes emprendedoras, actitudes emprendedoras y condiciones sociodemográficas y su incidencia en la variable dependiente., denominada como potencial emprendedor. Este planteamiento ha sido fuertemente criticado por varios académicos, al asegurar que el potencial emprendedor, no depende de las variables independientes previamente mencionadas, sino que de 2 aristas únicamente: la credibilidad/seguridad personal del potencial emprendedor (revisar la Figura 2), así como en la propensión a actuar/aversión al riesgo (Olmos, 2011).

Figura 1. Parametrización de las variables, adjuntas al Modelo de Potencial Emprendedor

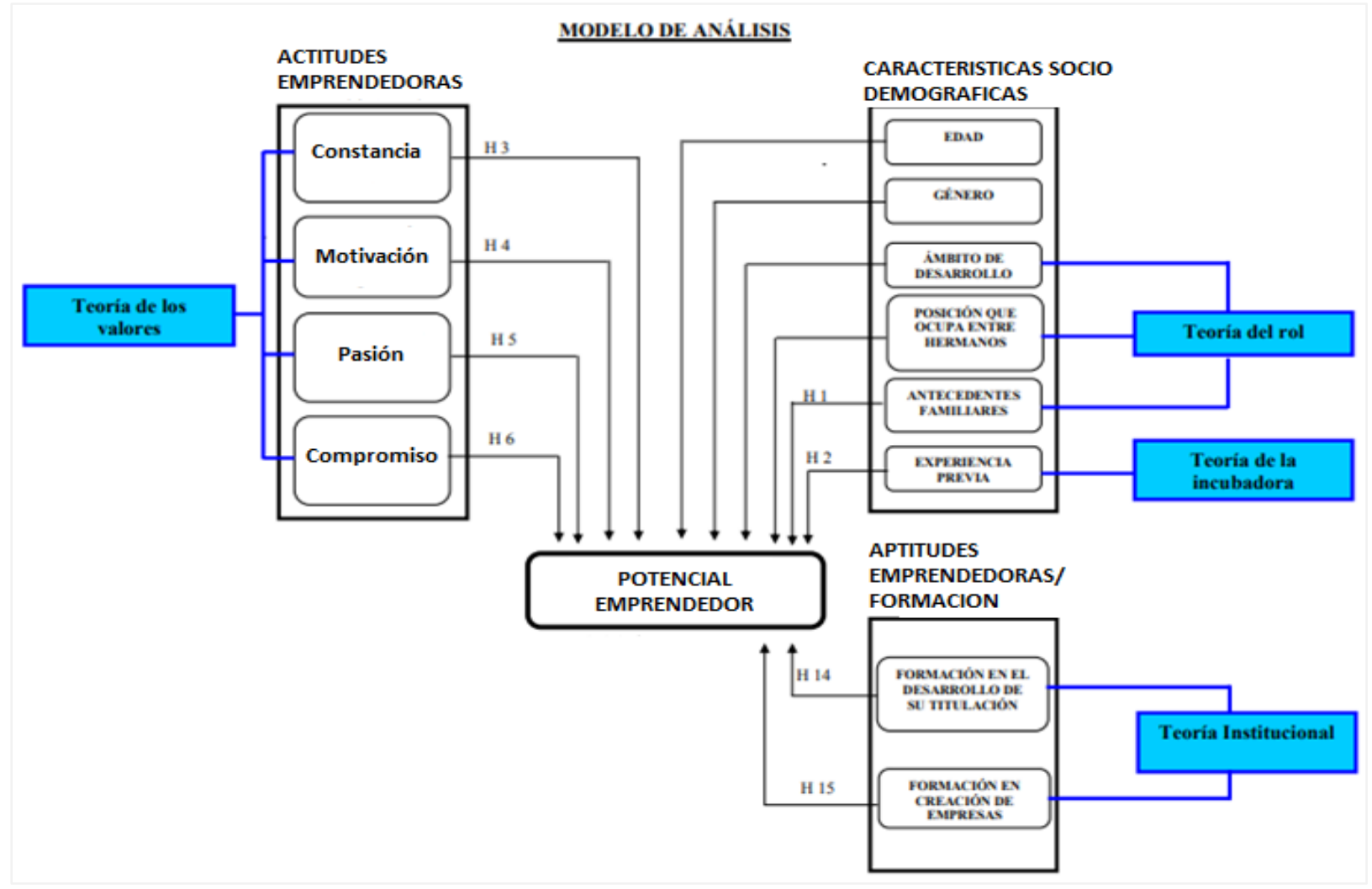

Fuente: Roberto E. Olmos, 2011, Universidad Complutense de Madrid Tesis Doctoral, Actitud Emprendedora en los Estudiantes Universitarios: Un Análisis de Factores Explicativos en la comunidad de Madrid. 
Figura 2. Modelo Alternativo de Variables que inciden sobre el Potencial Emprendedor

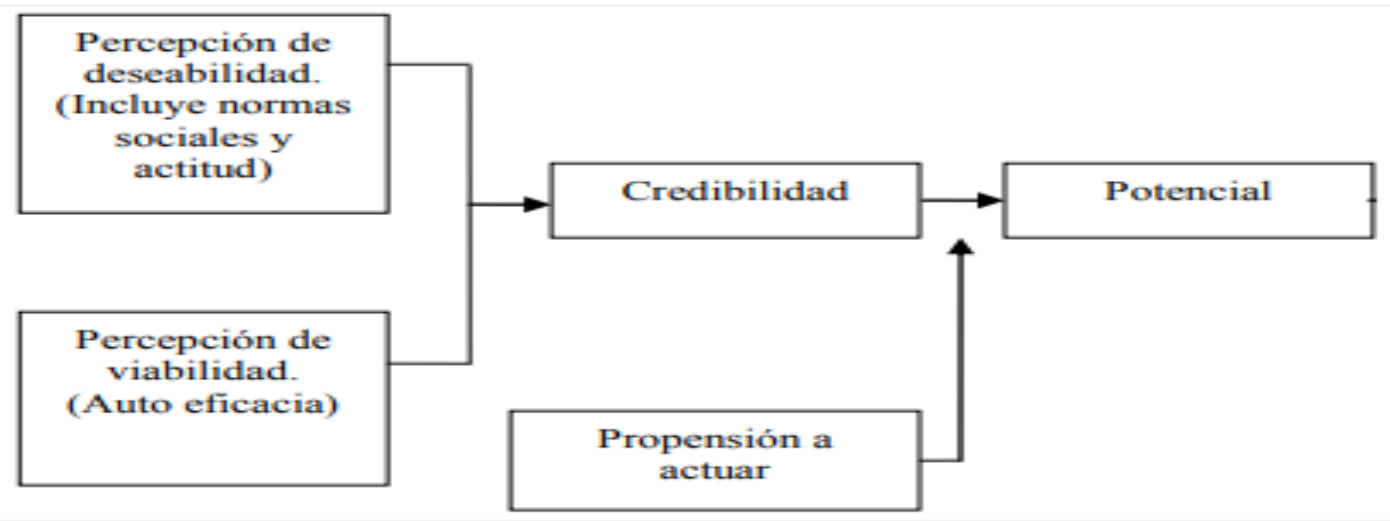

Fuente: Compilación Tesis Doctoral: Roberto E. Olmos de Krueger y Brazeal (1994)

Por otro lado, los postulados de los Doctores Krueger, Krauss y Brazeal, quienes señalan que son otros factores/variables las que llegan a incidir en el potencial emprendedor de las(os) estudiantes de la UNAH-FCEAC (Krauss, 2011), destacando entre estos:

- La propensión para actuar de los estudiantes, es decir el nivel de aversión al riesgo de fracasar con la iniciativa de negocios (revisar figura 2).

- La credibilidad del emprendedor(a) con el sistema que rige/predomina en los mercados, sobre los cuales se pretende instalar el modelo de negocios, a nivel de las garantías que facilita el estado para respaldar el marco legal y condiciones para la libre competencia, en igualdad de condiciones, además de la permeabilidad que presentan las barreras comerciales, impuestas por los competidores de "primera línea" /líderes en el sector (revisar Figura 2). Cabe mencionar, que además de lograr conceptualizar y normalizar el término de emprendimiento, es vital hacer mención, que el GEM, lo parametriza en distintos subtipos y/o dimensiones, que facilitan la labor de caracterizar y desarrollar los perfiles de emprendimiento que se gestan en distintos contextos. Lo enunciado con antelación, define a 8 categorías generales de emprendedores, según el GEM y distintos autores, como son:

1. Visionario: es aquel emprendedor que procura ir un paso adelante de las coyunturas $y$ tendencias, estableciéndose en rubros que estarán en auge, en el futuro.

2. Inversionista: es aquel emprendedor que explora y busca la máxima rentabilización de su dinero, en proyectos, que 
demuestran un riesgo menor, modelando distintos escenarios de factibilidad financiera previa a su gestión.

3. Especialista: el emprendedor especialista suele tener un perfil técnico, con extensiva experiencia $y$ conocimiento en un rubro determinado. $\mathrm{Y}$ aunque empieza un proyecto empresarial, sus conocimientos están muy centrados en el sector donde se especializa.

4. Persuasivo: es aquel tipo de emprendedor que suele liderar más que desarrollar el producto o servicio. Se encarga de mantener la convicción en su equipo.

5. Intuitivo: el emprendedor intuitivo sabe dónde está el negocio.

6. Emprendedor-empresario: este emprendedor ya sabe del mundo de la empresa. Nada le es nuevo. El empresario emprendedor asume el riesgo de emprender, pero a diferencia del intuitivo o el visionario, le gusta consolidar los proyectos, más que emprender.

7. Emprendedor-oportunista: este emprendedor sabe detectar las oportunidades de negocio y los pasos que debe seguir para gestionarla. Conoce ampliamente el mercado, sus bondades y las explota.

8. Emprendedor-vocacional: Es aquel emprendedor que entrelaza todos los perfiles anteriores, el cual fue formado y preparado para desarrollar, supervisar y acompañar emprendimientos.

Por consiguiente, la importancia de sensibilizar al estudiante universitario sobre la importancia de generar negocios y empleo es vital para su formación integral, al conocer y explorar sus fortalezas, (Argueta, Ortega, \& Acevedo, 2012).

A nivel regional, cabe destacar el rol que han desempeñado varias universidades latinomericanas, ubicadas en países como Chile, México y Brasil, Ecuador, al promover el emprendimiento desde dos trincheras (incubadoras de emprendimiento e incubadoras de ciencia), resaltando así, la trascendencia de la academia en la formación integral del futuro profesional. Destacando un análisis del Global Entrepreneurship Research Association (GERA) que, en el año 2012, estudio a las incubadoras de emprendimiento, desde dos sub tipos de aristas: Emprendimiento por Necesidad y Emprendimiento por Oportunidad. En esta pesquisa, realizada por el GERA, la potencia económica regional, que más le está apostando a la sensibilización, formación y facilidades de capital semilla para la creación 
de nuevos negocios, es la chilena, al enfocarse en la creación de Pymes, en la rama tecnológica, revisar Figura 3 a continuación:
De igual manera, se destacan los esfuerzos de las universidades ecuatorianas, para fomentar la creación de negocios, emulando el modelo chileno (revisar la figura 4).

Figura 3. Proceso de Emprendedores Nacientes

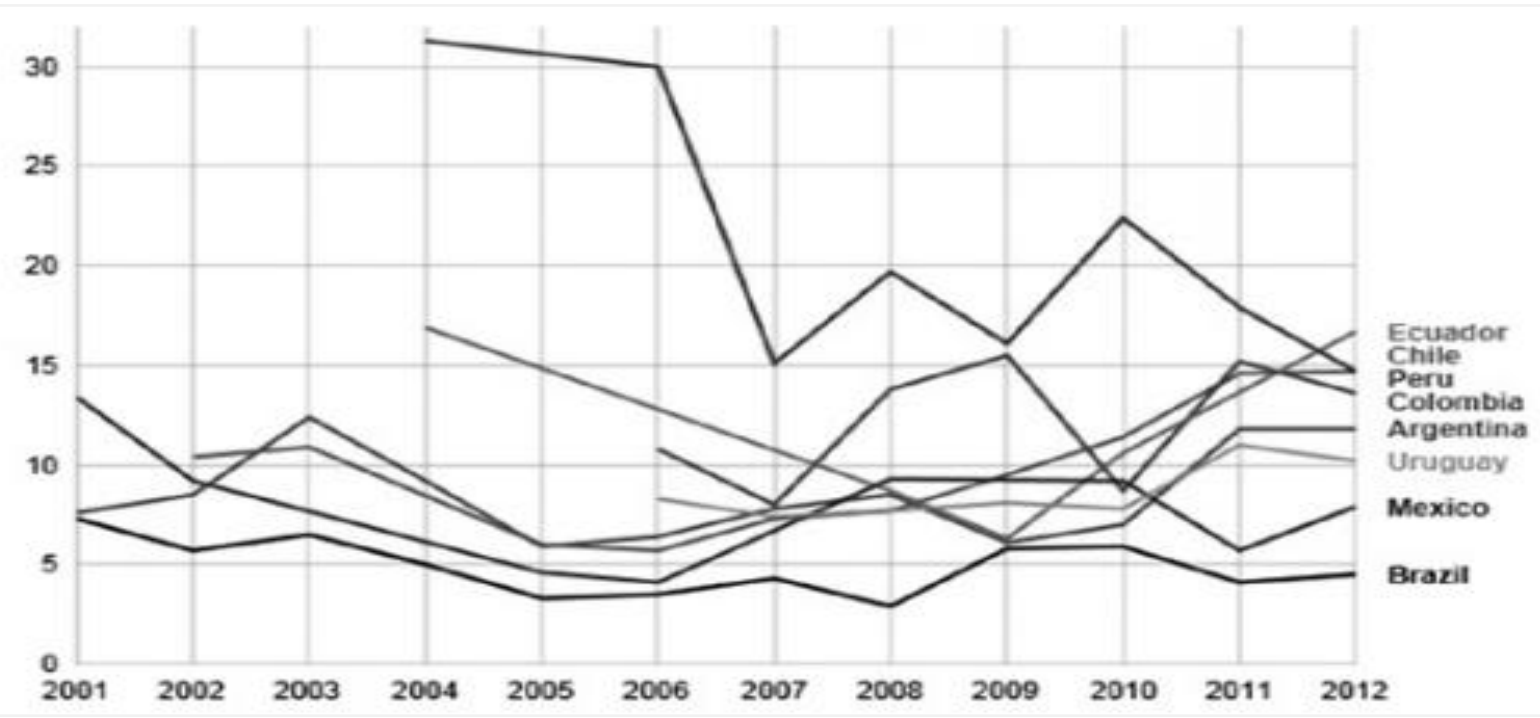

Fuente: Global Entrepreneurship Research Association (GERA) 2012

Figura 4. Actividad de Emprendimiento por Identificación de Oportunidad

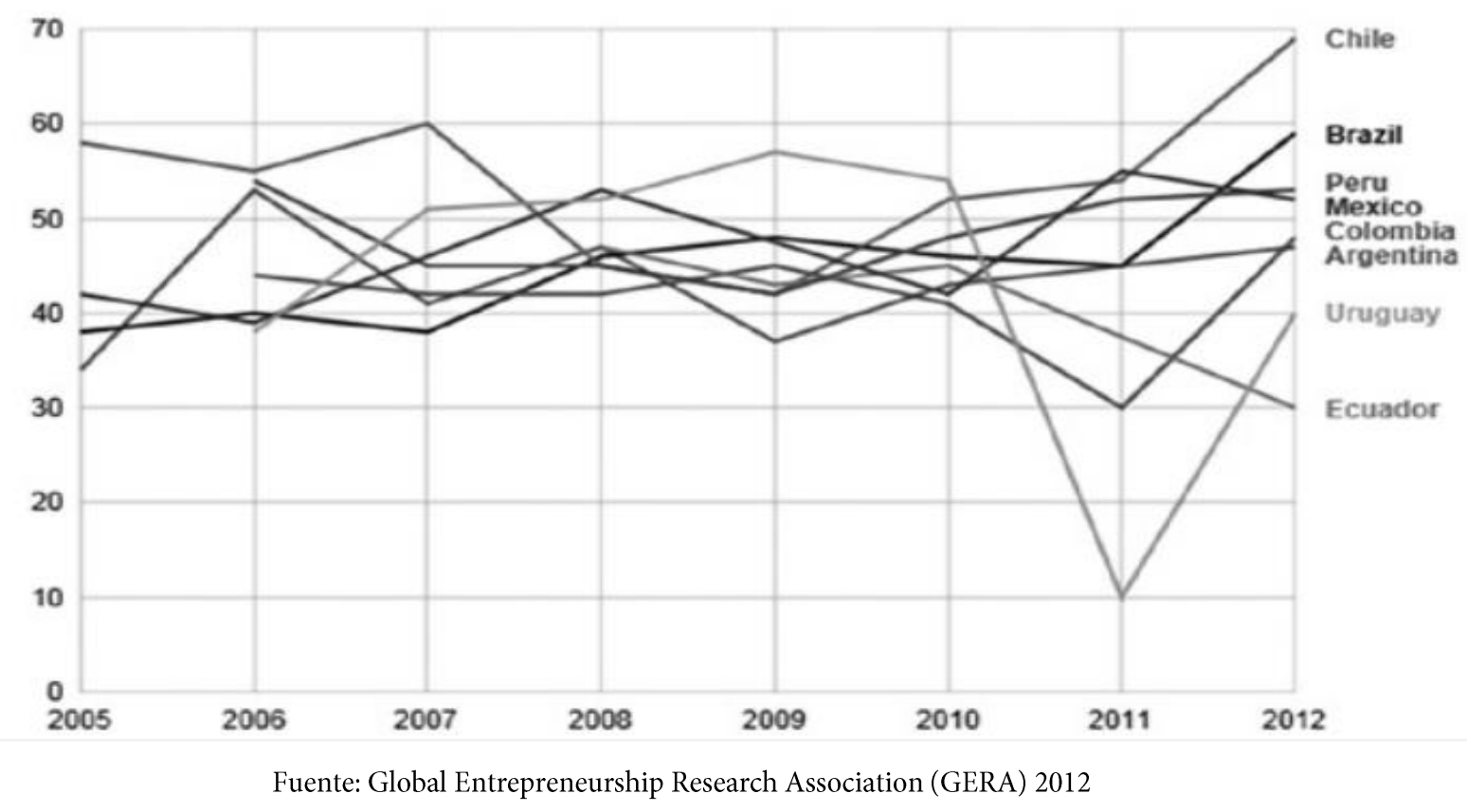


Luego de analizar la figura 4, amerita señalar que el apoyo técnico, sin lugar a duda, estimula el cálculo del riesgo de inversión. Mitigando el fenómeno de emprender sin información y de manera empírica, de los estudiantes universitarios, al permitir un robustecimiento de sus habilidades, competencias y pericias, en materia de marketing, gestión de operaciones y financieras, para articular los escenarios optimistas, pesimista y más probable.
Por otro lado, es vital el hacer mención del esfuerzo realizado por las instituciones de educación superior ecuatorianas, en materia de sensibilización, quienes ya han cosechado frutos en el combate del fenómeno del desempleo, al formar a sus estudiantes de pre y posgrado, con las herramientas y motivación necesaria, para iniciar con sus proyectos de autoempleo. Favor revisar la figura 5, a continuación:

Figura 5. Actividades de Emprendimiento por Necesidad

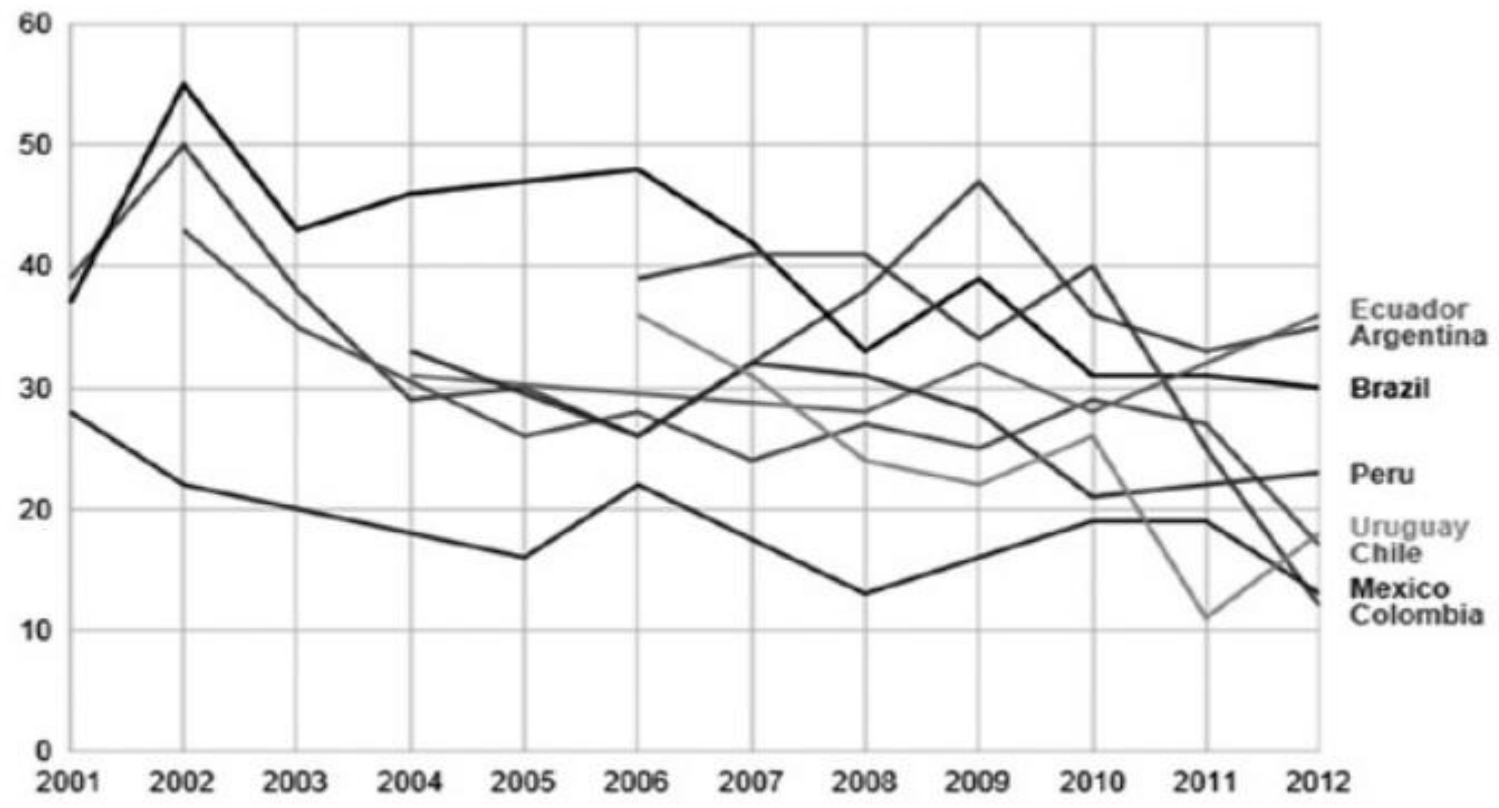

Fuente: Global Entrepreneurship Research Association (GERA) 2012

Es así que, al contar con un pleno conocimiento de las realidades sociales y macroeconómicas de un país, varias instituciones de educación superior en la región se han orillado por el estímulo a la formación de jóvenes emprendedores.
3. METODOLOGÍA Y FUENTE DE DATOS

Este trabajo investigativo, de tipo cualitativo, desarrollo la descripción del perfil emprendedor (desde una óptica cualitativa), de las y los jóvenes millennials universitarios 
de la FCEAC, a través del estudio de las 9 dimensiones de emprendimiento y la caracterización de sus necesidades, condicionantes, fortalezas y debilidades en materia de su potencial emprendedor (Sabino, 1994).

La pesquisa en mención, en su marco cualitativo, procuro estudiar las variables: actitudes emprendedoras, aptitudes emprendedoras y potencial emprendedor, a través de la aplicación de dos técnicas cualitativas, como ser: la entrevista semiestructurada y observación de las y los estudiantes de la FCEAC, en el 2ndo periodo del año 2019 (Bernal, 2015).

Cabe mencionar, que la aplicación de las entrevistas semiestructuradas y observación de las y los estudiantes de la FCEAC (la unidad de análisis para esta pesquisa), contemplo la participación de todas las carreras adscritas a dicha facultad, sin embargo, debido a algunas limitantes, el estudio solamente evaluó a estudiantes pertenecientes a las carreras de: Mercadotecnia, Finanzas y Administración de Empresas. De manera que, de los 26 estudiantes observados y entrevistados, el $50 \%$ de ellas(os), pertenecen a la carrera de Admón. de Empresas, 25\% a la carrera de Mercadotecnia y $25 \%$ pertenecen a la carrera de Finanzas. Un elemento para señalar es que previo a la aplicación de las técnicas cualitativas sobre los 26 estudiantes en mención, se tenía contemplado entrevistar a alrededor de 50 estudiantes, sin embargo, al estudiar a 26 de ellas(os) se alcanzó la saturación teórica, entre otros.

Los modelos utilizados para la primera y segunda fase de este análisis emanan de los insumos propuestos y aplicado en la Universidad Complutense de Madrid (UCM) en el año 2017, los cuales ya fueron validados previamente y requirieron únicamente de algunos ajustes al contexto hondureño (Freire, 2017).

\section{ANÁLISIS Y DISCUSIÓN DE RESULTADOS}

Luego de levantar varios insumos de fuentes primarias (entrevistas y observación) y secundarias (exploración teórica y empírica), para el desarrollo de esta primar fase, es importante el recorrer, el apéndice A, figura A1 y tabla A1 y A2, cuyos desarrollos, resumen los hallazgos encontrados, tras la aplicación de dos herramientas de orden cualitativo, sobre los estudiantes en mención.

En este apartado, vital el destacar, que las y los estudiantes de la FCEAC, presentan un alto potencial emprendedor, al considerar el alto componente actitudinal entre las(os) mismas(os), articulado con una alta propensión a innovar. Otro elemento, que favorece a las(os) estudiantes de la FCEAC a emprender, es el robustecimiento de las 
competencias investigativas, que les permite, diagnosticar una realidad y descomponerla en varios escenarios de inversión.

Para concluir con este apartado, es válido el inferir que las jóvenes estudiantes, presentan un Potencial Emprendedor superior al de los jóvenes estudiantes, al presentar una menor aversión al riesgo y mayor motivación, en marco de parámetros similares, a los realizados por la tesis de la Doctora María Teresa Freire, en el año 2017, aplicado en la masa estudiantil de la Universidad Complutense de Madrid (Freire, 2017).

\section{CONCLUSIONES}

Luego de explorar los insumos teóricos y empíricos, en el marco de la temática del Potencial Emprendedor, a nivel de las y los estudiantes universitarios, en la región latinoamericana, así como en el país (de forma particular en aquellos adscritos a la Facultad de Ciencias Económicas, Administrativas y Contables de la Universidad Nacional Autónoma de Honduras, en ciudad universitaria, Tegucigalpa). Es válido el denotar, que dentro de las principales características que ellas y ellos presentan, destacan:

Los Estudiantes en la región latinoamericana, están fuertemente influenciados por dos corrientes de pensamiento emprendedor: El
Emprendimiento por Necesidad, así como el Emprendimiento por Oportunidad.

Destacando, que los estudiantes emprendedores latinoamericanos del Ecuador dominan el ámbito del emprendimiento por necesidad en la región, al generar sus propios ingresos, en modelos de negocios que procuran innovar en mercados altamente competitivos.

Así mismo, es importante hacer alusión a los esfuerzos encaminados por los estudiantes emprendedores chilenos, que se decantan por la gestión de modelos de negocios por oportunidad, en el ámbito de las nuevas tecnologías de la información.

De igual manera, al trasladar el análisis del Potencial Emprendedor al contexto local, la pesquisa demostró, que aquellos estudiantes, con edades entre 22-28 años, pertenecientes a la FCEAC, presentan menos aversión al riesgo de emprender, además de ubicar sus inquietudes emprendedoras, en el ámbito de los sectores de Alimentos y Bebidas, Servicios Profesionales y Turismo por oportunidad.

Por otro lado, es vital el mencionar que, al escudriñar las principales causales de tipo exógeno, que condicionan el Potencial Emprendedor (PE) de las y los estudiantes de la FCEAC, al momento de emprender: destacan: 
- Acceso a fuentes de financiamiento $\mathrm{y} / \mathrm{o}$ capital semilla, suficiente para iniciar con la propuesta de negocio.

- Asesoría técnica en la incubación y desarrollo de sus iniciáticas de negocio.

- Alta burocratización en la inscripción de las empresas.

De igual manera, dentro de las principales causales de tipo endógeno, que condicionan/limitan el PE de las y los estudiantes de la FCEAC, son:

Acceso y dominio de las tecnologías de la información, en materia de equipo para asegurar la calidad de los alimentos, software de control administrativo y contable, entre otros.

Dentro de otros puntos a robustecer, es el "networking", que permita a las y los estudiantes, para fortalecer la cadena de valor y suministro, así como las posibles alianzas estratégicas para sus negocios.

Más allá de las condicionantes/limitantes que regulan el Potencial Emprendedor (PE), de las y los estudiantes de la FCEAC, es importante el señalar existen 2 factores que desde la UNAH debe de seguir promoviendo, desde un enfoque de Potencial Emprendedor:

1. Las actitudes emprendedoras

2. Las aptitudes orientadas al emprendimiento.
Finalmente, cabe mencionar que, en la rama actitudinal, los estudiantes conciben que se deben de fortalecer los elementos de constancia y compromiso emprendedor, así como de insumos tecnológicos, desde la academia.

\section{REFERENCIAS}

Adler, A. (1924). "Práctica y teoría de la psicología del individuo”. Barcelona: Paidós.

Argueta, J., Ortega, C., \& Acevedo, M. (2012). Observatorio Economico y de Emprendimiento. Ciencia y Tecnologia, 1323.

Baumoul, W. (2002). The free-market innovation machine: analyzing thegrowth miracle of capitalism. Princeton University Press, Pg 336.

Bernal, C. (2015). Metodologia de la Investigación 3era Edición. Mexico: Pearson.

Cervilla, M., \& Puente, R. (2013). Modelos de Negocio de Emprendimiento por y para la base de la Piramide. Revista de Ciencias Sociales, Pgs 209-308.

COHEP. (2019). Perfil del Millenial en Honduras 2019. Tegucigalpa, Francisco Morazán, Honduras: Delco Marketing.

David, D., \& Posada, E. E. (12 de Abril de 2019). Milenials en Honduras. El 79\% de los Millennials del país ganan menos de 15,000 Lempiras, pág. 15. 
FOSDEH. (2018). Informe Indicadores Macro

Economicos HN 2018. Tegucigalpa, Francisco

Morazan, HN: Foro Social de la Deuda

Externa y Desarrollo de Honduras.

Freire, M. (2017). Tesis Doctoral. Formación para el Emprendimiento en la Enseñanza de Economía y Escuelas de Negocios. Madrid, España: Facultad de Ciencias Economicas y Empresariales, Departamento de Historia e Instituciones Economicas. Obtenido de https://eprints.ucm.es/40779/1/T38236.pdf

GEM. (24 de 05 de 2019). Global Entepreneurship Monitor. Obtenido de https://gem.ufm.edu/que-es-gem/

Krauss, C. (2011). Actitudes emprendedoras de los estudiantes universitarios:El caso de la Universidad Católica del Uruguay*. Dimens. empres, págs. 28-40.

Olmos, R. (10 de 06 de 2011). Actitud Emprendedora en los Estudiantes
Universitarios: Un Análisis de Factores Explicativos en la comunidad de Madrid. Tesis Doctoral. Madrid, España: Facultad de Economia, UCM.

Robbinson, P. (1991). An attitude approach to the prediction of entrepreneurship. Entrepreneurship: Theory \& Practice. 13.

Sabino, C. (1994). Como hacer una Tesis y Redactar Trabajos Cientificos. Caracas, Venezuela: Panapo.

Sparano, H. (2014). Emprendimiento en America Latina y su Impacto en la Gestión de Proyectos. Revista Dimensión Empresarial, Vol No 12(No 2), Pgs. 95-106.

Venecian, J. (1989). Características del empresario en España. Papeles de Economía Española, num. 39/40.

\section{APENDICE A}

Figura A1. Diagrama Sagital de las Variables en Estudio

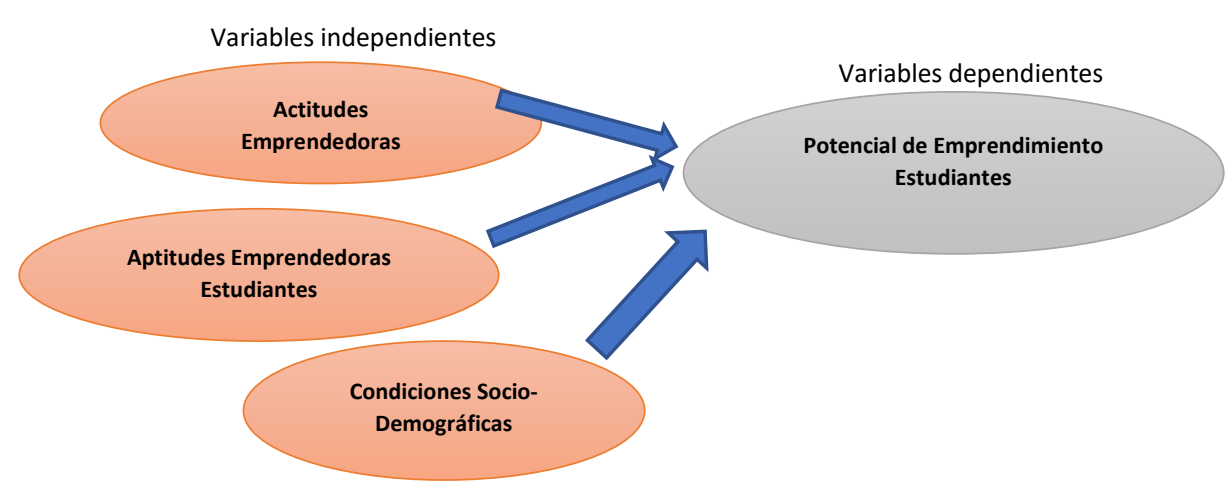

Fuente: Interpretación Personal del Fenómeno 
Revista Economía y Administración Vol. 10, N.2, 2019

Tabla A1. Preguntas Semi Estructuradas Aplicadas en el Estudio

\begin{tabular}{|c|c|c|}
\hline No Pregunta & Descripción de la Pregunta & Respuesta Generalizada, acotada en palabras claves \\
\hline Pregunta No 1 & $\begin{array}{l}\text { ¿Cómo describiría las condiciones actuales de } \\
\text { inversión en el país? }\end{array}$ & $\begin{array}{l}\text { Inestabilidad Jurídica, Alta Riesgo de Inversión, Extorsión, } \\
\text { Inestabilidad Política, Alto Riesgo de Gestión debido } \\
\text { manifestaciones, Mano de Obra con altas necesidades de } \\
\text { capacitación, Infraestructura limita la Logística y } \\
\text { Aprovisionamiento. }\end{array}$ \\
\hline Pregunta No 2 & $\begin{array}{l}\text { ¿Cuáles considera que son los principales retos } \\
\text { que afrontan los empresarios hoy en día? }\end{array}$ & $\begin{array}{l}\text { Alto Intereses para obtener un Apalancamiento de } \\
\text { Financiero, Altas Tasas Tributarias, Alta Burocratización } \\
\text { para la Gestión de los Permisos de Operación, Poco Apoyo } \\
\text { en el Capital Semilla, Extorsión, Competencia Desleal. }\end{array}$ \\
\hline Pregunta No 3 & $\begin{array}{l}\text { ¿Qué elementos consideraría, deberían de estar } \\
\text { presentes, en el perfil ideal de un emprendedor en } \\
\text { la actualidad? }\end{array}$ & $\begin{array}{l}\text { Capacidad para Planificar, Controlar y Ejecutar Proyectos } \\
\text { de Inversión, Competencias Tecnológicas Básicas, } \\
\text { Competencias de Comunicativas y de Venta, } \\
\text { Competencias Estadísticas (Realizar Proyecciones y } \\
\text { Modelar Escenarios) }\end{array}$ \\
\hline Pregunta No 4 & $\begin{array}{l}\text { ¿Qué opciones de inversión ha considerado, en el } \\
\text { corto, mediano o largo plazo? Si no lo ha hecho, } \\
\text { favor explicar porque No }\end{array}$ & $\begin{array}{l}\text { Sector Alimentos y Bebidas, Sector Turismo, Sector } \\
\text { Servicios }\end{array}$ \\
\hline Pregunta No 5 & $\begin{array}{l}\text { ¿Qué elementos en su Formación } \\
\text { Profesional/Aptitudinal, le han ayudado a } \\
\text { entender de mejor manera el mercado de } \\
\text { negocios en el cual desea incursionar? Explique } \\
\text { cómo le han apoyado estos insumos }\end{array}$ & Competencias Estadísticas, Habilidades Investigativas \\
\hline Pregunta No 6 & $\begin{array}{l}\text { ¿Cuáles son las principales Actitudes } \\
\text { Emprendedoras que le caracterizan como } \\
\text { futura(o) emprendedor, para encaminar y } \\
\text { concretar sus propuestas de } \\
\text { negocio/empresariales? Explique el porqué de } \\
\text { cada una }\end{array}$ & Constancia, Innovación, Motivación \\
\hline Pregunta No 7 & $\begin{array}{l}\text { De las actitudes y aptitudes previamente } \\
\text { mencionadas ¿Cuáles de las previamente } \\
\text { mencionadas, deberían de ser reforzadas desde la } \\
\text { academia? }\end{array}$ & $\begin{array}{l}\text { Habilidades Investigativas (Inteligencia de Mercados) e } \\
\text { Innovación (a través de la Tecnología). }\end{array}$ \\
\hline Pregunta No 8 & $\begin{array}{l}\text { Considera la opción de emprender, en el corto, } \\
\text { mediano o largo plazo, Porque? Explique el } \\
\text { porqué de su elección. (Si su respuesta fue no, } \\
\text { muchas gracias por atender a la entrevista) }\end{array}$ & $\begin{array}{l}\text { El emprendimiento se deberá de manifestar en el largo } \\
\text { plazo, evitando las poco flexibles opciones de } \\
\text { apalancamiento financiero, que oferta la banca comercial. }\end{array}$ \\
\hline Pregunta No 9 & $\begin{array}{l}\text { ¿Conoce de alguna institución, que le podría } \\
\text { brindar apoyo financiero/técnico en el marco de } \\
\text { sus iniciativas de emprendimiento? }\end{array}$ & $\begin{array}{l}\text { Más allá de la banca privada, los estudiantes, no conocen } \\
\text { muchas opciones para recibir apoyo financiero/técnico } \\
\text { para concretar y robustecer sus modelos de negocios. }\end{array}$ \\
\hline
\end{tabular}

Fuente: Interpretación Personal, Modelo de Roberto Olmos, ajustada al contexto local. 
Tabla A2. Observaciones Compiladas de los Estudiantes en espacios Académicos

\begin{tabular}{|c|c|c|}
\hline$\underline{\text { Variables }}$ & Dimensiones & Observaciones Registradas \\
\hline \multirow{2}{*}{$\begin{array}{l}\text { Aptitudes } \\
\text { Emprendedoras }\end{array}$} & $\begin{array}{l}\text { Formación en el Desarrollo de su } \\
\text { Titulación }\end{array}$ & $\begin{array}{l}\text { Poca articulación de las Aptitudes en el Desarrollo de la Titulación } \\
\text { con la gestión emprendedora }\end{array}$ \\
\hline & $\begin{array}{l}\text { Formación en el Desarrollo de su } \\
\text { Capacidad Emprendedora }\end{array}$ & $\begin{array}{l}\text { Se percibe escasa formación en las Aptitudes de la Capacidad } \\
\text { Emprendedora de los Estudiantes }\end{array}$ \\
\hline \multirow{4}{*}{$\begin{array}{l}\text { Actitudes } \\
\text { Emprendedoras }\end{array}$} & Constancia & \multirow{4}{*}{$\begin{array}{l}\text { Las Actitudes que predominan es la Motivación, así como la Pasión } \\
\text { por sus iniciativas de emprendimiento, entre las y los estudiantes de } \\
\text { la FCEAC.UNAH }\end{array}$} \\
\hline & Pasión & \\
\hline & Motivación & \\
\hline & Compromiso & \\
\hline \multirow{4}{*}{$\begin{array}{c}\text { Características } \\
\text { Sociodemográficas }\end{array}$} & Edad & Edades entre 22-26 años de edad \\
\hline & Genero & $65 \%$ Mujeres 35\% Hombres \\
\hline & Carrera en la FCEAC-UNAH-CU & 50\% Admón. Empresas, 25\% Merca, 25\% Finanzas \\
\hline & Experiencia Previa & Solamente el 7\% de ellas(os) contaba con alguna experiencia laboral \\
\hline \multirow{3}{*}{$\begin{array}{c}\text { Potencial } \\
\text { Emprendedor }\end{array}$} & ALTO & \multirow{3}{*}{$\begin{array}{l}\text { Pese a contar con varias limitantes a nivel de sus Aptitudes } \\
\text { Emprendedoras, en el ámbito de su Capacidad Emprendedora, el PE, } \\
\text { percibido es alto, con la particularidad de Emprendimiento por } \\
\text { Necesidad, debido a las condiciones del Mercado Laboral y sus } \\
\text { Comentarios en la Entrevista }\end{array}$} \\
\hline & Medio & \\
\hline & Bajo & \\
\hline
\end{tabular}

Fuente: Interpretación Personal, técnica de observación, estudiantes FCEAC-UNAH. 